\title{
Rayleigh-Taylor instability under curved substrates: An optimal transient growth analysis
}

\author{
Gioele Balestra, ${ }^{1, *}$ P.-T. Brun, ${ }^{2}$ and François Gallaire ${ }^{1}$ \\ ${ }^{1}$ Laboratory of Fluid Mechanics and Instabilities, EPFL, CH1015 Lausanne, Switzerland \\ ${ }^{2}$ Department of Mathematics, Massachusetts Institute of Technology, Cambridge, Massachusetts 02139, USA
}

(Received 18 February 2016; published 7 December 2016)

\begin{abstract}
We investigate the stability of thin viscous films coated on the inside of a horizontal cylindrical substrate. In such a case, gravity acts both as a stabilizing force through the progressive drainage of the film and as a destabilizing force prone to form droplets via the Rayleigh-Taylor instability. The drainage solution, derived from lubrication equations, is found asymptotically stable with respect to infinitesimally small perturbations, although in reality, droplets often form. To resolve this paradox, we perform an optimal transient growth analysis for the first-order perturbations of the liquid's interface, generalizing the results of Trinh et al. [Phys. Fluids 26, 051704 (2014)]. We find that the system displays a linear transient growth potential that gives rise to two different scenarios depending on the value of the Bond number (prescribing the relative importance of gravity and surface tension forces). At low Bond numbers, the optimal perturbation of the interface does not generate droplets. In contrast, for higher Bond numbers, perturbations on the upper hemicircle yield gains large enough to potentially form droplets. The gain increases exponentially with the Bond number. In particular, depending on the amplitude of the initial perturbation, we find a critical Bond number above which the short-time linear growth is sufficient to trigger the nonlinear effects required to form dripping droplets. We conclude that the transition to droplets detaching from the substrate is noise and perturbation dependent.
\end{abstract}

DOI: 10.1103/PhysRevFluids.1.083902

\section{INTRODUCTION}

The interface between two fluids might become unstable if the heavier fluid accelerates towards the lighter one. The resulting instability, known as the Rayleigh-Taylor instability (RTI), is found across a broad range of scales in nature and industrial settings [1-3]. Everyday examples include the formation of droplets in a film of condensed vapor underneath a lid or below the ceiling of a pool.

Avoiding, or enhancing, the formation of droplets is of paramount importance in several engineering situations. In coating problems, the RTI can lead to undesired irregularities, deteriorating the uniformity of the final product. Likewise, the RTI could jeopardize the use of liquid metals as plasma-facing materials coated on the inside of toroidal fusion reactors [4]. Droplets detaching from the reactors' wall could fall in the plasma and quench the process. A response to this specific concern has recently been brought, revealing the stabilizing effect of the substrate curvature [5]. This salutary feature must have been known empirically by architects for centuries, as one can infer from the curved roofs of traditional hammams, presumably designed in a way to prevent condensed vapor to drip from the ceiling onto clients. Note that similar stabilizing effects also play a central role in the fabrication of thin polymeric shells or chocolate eggs [6]. In other contexts, a variety of stabilizing strategies, including the use of heat and electrical current, have been studied (see Refs. [7-10], for example).

In the laboratory, the RTI may be obtained using a thin layer of oil coated on the underside of a horizontal substrate. In this case, the droplets are found to arrange in well-defined lattices [11]. In fact, the film interface is always linearly unstable and the most amplified wavelength (proportional

*gioele.balestra@epfl.ch 
to the capillary length) sets the properties of the observed patterns. For films much thinner than the capillary length, static pendant drops can form, translate, and collide [12,13]. When inclining the solid substrate, the RTI is marginally modified for small slopes, but can be annihilated if the slope is large enough [14]. The distinction between these two regimes can be rationalized using an absolute/convective instability analysis $[14,15]$. Similarly, when the inclination of the substrate varies uniformly, i.e., the solid surface has a finite curvature, Trinh et al. [5] showed that the Rayleigh-Taylor instability can be suppressed for a thin film if surface tension forces are strong enough. Gravity acts both as a stabilizing force originating in the progressive drainage of the film and as a destabilizing force prone to form dripping droplets. These components vary along the substrate, making the search for a criteria delicate.

Trinh et al. [5] performed a linear stability analysis for an axially invariant film coated on the inside of a horizontal cylinder, restricting their work to the most unstable region near the top of the cylinder. There, they showed that the only significant parameter was a modified Bond number prescribing the ratio between gravitational and surface tension forces in this geometry. They found a critical value of order 1 for this dimensionless number. Above this threshold, perturbations display an initial transient growth, and, below this critical value, all perturbations decay monotonically. Their experiments confirmed this trend for sufficiently small Bond numbers. For higher values the physical picture is more complex. In particular, the transition between droplets that form but do not detach from the substrate and droplets that form and pinch off takes place at a significantly higher Bond number (of order 100), which is not accounted for by theory. Here we show that these results can be rationalized by an optimal transient growth analysis for such a thin film when considering the entire circular geometry, finite times, and finite film thicknesses, thereby generalizing the approach of Trinh et al. [5].

Time-independent flows are defined as linearly stable in the asymptotic limit of large times if all eigenvalues are in the stable complex half-plane. Yet, these flows might experience a transient growth at short times, if their dynamics is governed by a non-normal operator. In fluid dynamics, many operators have a set of nonorthogonal eigenfunctions and can therefore display a large amplification of the energy contained in the initial condition. The transient growth might be sufficient to trigger the transition to the bifurcated state. Nonmodal approaches have been successful in understanding the transition mechanisms at play in various wall-bounded shear flows (see Ref. [16] for a review). As an example, in the context of liquid films, Bertozzi and Brenner [17] showed that the transient growth is responsible for the initiation of the finger instability in an asymptotically linearly stable configuration. More recently, Gallino et al. [18] found that an asymptotically stable rising droplet forms an instability at the tail for a lower perturbation amplitude when the initial shape is close to the one obtained by the optimal transient growth analysis.

Here we apply this tool to the RTI under a curved substrate. In contrast to the aforementioned studies, the base flow is time dependent, which rules out any classical eigenvalue calculation. However, a nonmodal approach is suitable [16]. The optimal initial conditions maximizing the gain are computed on the entire physical space for several time horizons, Bond numbers, and finite film thicknesses. These calculations allow for a better understanding of the most amplified perturbations yielding the droplet formation. Two regimes are evidenced. For surface-tension-dominated flows, the optimal perturbations are fronts and their maximal gain is achieved when located at the equator. When gravity dominates surface tension, optimal perturbations correspond to oscillations at the north pole, possibly resulting in droplets. Indeed, large-Bond-number regimes display a strong transient growth of perturbations which, in turn, might trigger the nonlinear effects at the origin of the droplet formation and dripping observed in experiments. Using nonlinear simulations, we show that depending on the initial perturbation amplitude, there indeed exists a critical Bond number above which dripping droplets start to occur.

The paper is structured as follows. Section II A presents the governing equations of the problem, the transient growth analysis is explained in Sec. II B, and the employed numerical methods are shown in Sec. II C. The results are presented in Sec. III. The two regimes are introduced in Sec. III A, and their dependence on the Bond number and on the film aspect ratio are described in Secs. III B 
and III C, respectively. The transient growth analysis over the entire geometry is discussed and compared to the one at the north pole in Sec. IV A. Nonlinear simulations are discussed in Sec. IV B. Conclusions are drawn in Sec. V.

\section{GOVERNING EQUATIONS AND METHODS}

\section{A. Lubrication and linear perturbation equations}

The inside of a cylindrical substrate of inner radius $R$ is coated with a viscous film of dimensionless thickness $\bar{H}(\theta, t)$ (using the initial average film thickness $H_{i}^{*}$ as a gauge), as depicted in Fig. 1(a). The drainage of this film occurs over a typical time $\tau_{d}=\mu R /\left(\rho g H_{i}^{* 2}\right)$, where $\mu$ is the dynamic viscosity, $\rho$ is the density, and $g$ is the gravitational field. Owing to the small aspect ratio and the typically large viscosity of the fluid used in such coatings, we model the flow using lubrication equations [19]. From the continuity equation one may show that the flow velocity is essentially one-dimensional (in the polar direction $\theta$, tangent to the substrate). Integrating the Stokes equations along the radial direction with the proper set of boundary conditions and considering mass conservation, the following lubrication equation is obtained (see Appendix A for the complete derivation):

$$
\bar{H}_{t}+\frac{1}{3}\left\{\bar{H}^{3}\left[\frac{\delta^{2}}{\mathrm{Bo}}\left(\bar{H}_{\theta \theta \theta}+\bar{H}_{\theta}\right)+\delta \bar{H}_{\theta} \cos \theta+\sin \theta\right]\right\}_{\theta}=0
$$

where indices represent partial derivatives. The modified Bond number representing the ratio between gravitational and surface tension forces, incorporating also geometric parameters, is given by $\mathrm{Bo}=$ $\rho g H_{i}^{*} R / \gamma$, where $\gamma$ is the surface tension. The second dimensionless parameter of the problem is the aspect ratio of the film, defined as $\delta=H_{i}^{*} / R$. Note that only the leading order terms of the curvature are kept.

The film-thickness evolution has to be computed numerically (see Sec. II C for details) since there is no analytical solution of Eq. (1) for finite aspect ratios $\delta$ and Bond numbers Bo. The solution resulting from an initially uniform film profile is shown in Fig. 1(b). As will become clear later, this solution is asymptotically linearly stable and we will refer to it as drainage solution $H(\theta, t)$. In the limit of vanishing aspect ratios, $\delta \ll 1$, and negligible surface tension effects, Bo $\gg \delta^{2}$, we can show that Eq. (1) has an approximate solution of the form $\left(1+\theta^{2} / 16\right) / \sqrt{1+2 t / 3}$ close to the

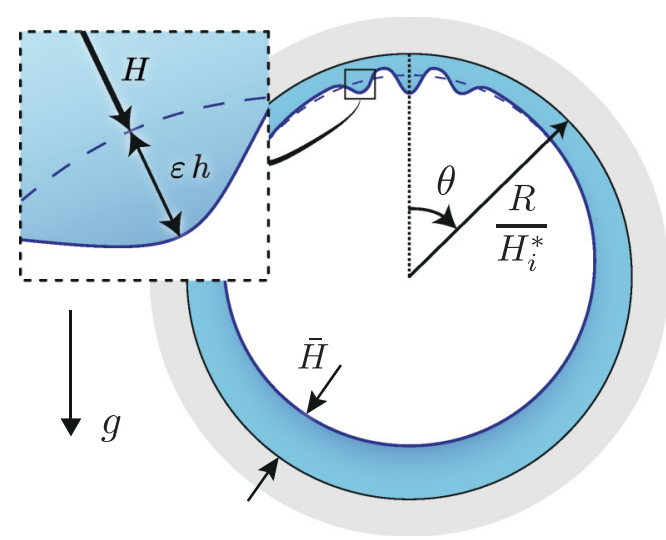

(a)

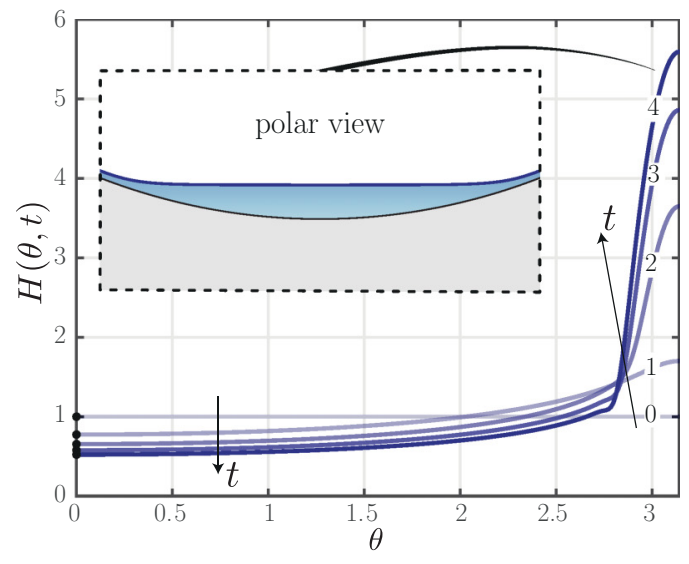

(b)

FIG. 1. (a) Sketch of the two-dimensional problem geometry. Inset: decomposition of film thickness $\bar{H}$ into draining solution $H$ and first-order perturbations $\varepsilon h$. (b) Evolution of the drainage solution $H$ at several times. The black bullets on the left represent the analytical solution at $\theta=0, H(0, t)=(1+2 t / 3)^{-1 / 2}$. Bo $=20$ and $\delta=10^{-2}$. Inset: fluid profile at the bottom of the cylinder for $t=4$. 


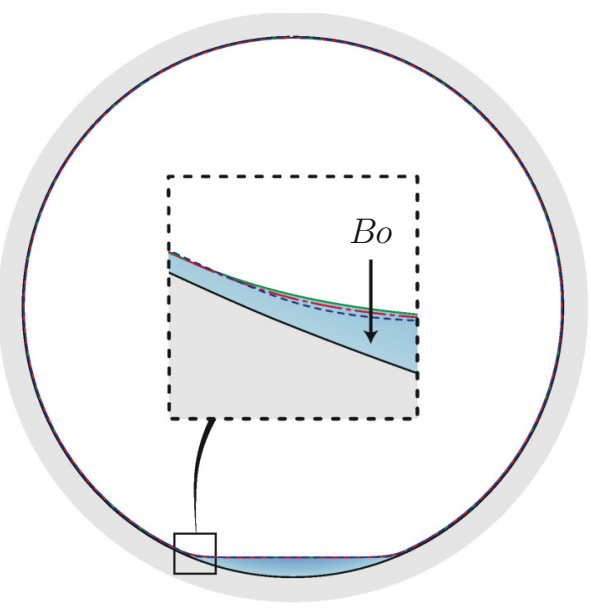

(a)

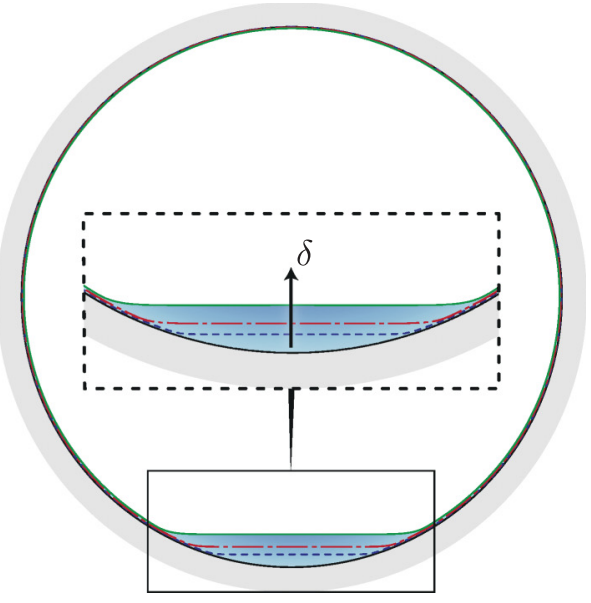

(b)

FIG. 2. Effect of the Bond number (a) and of the film aspect ratio (b) on the draining solution $H$ at the time $t=10$. (a) $\mathrm{Bo}=5,10$, and 50 for $\delta=10^{-2}$, (b) $\delta=5 \times 10^{-3}, 10^{-2}$, and $2 \times 10^{-2}$ for Bo $=20$.

north pole [6,20] and a similarity solution for the upper hemicircle, following Couder et al. [21]. As seen in Fig. 1(b), the analytical solution for $\theta=0$ is in good agreement with the numerical one also for finite $\delta$ and Bo values.

In order to obtain the linear perturbation equation necessary for the transient growth analysis, we decompose the film thickness as [see inset of Fig. 1(a)]:

$$
\bar{H}(\theta, t)=\underbrace{H(\theta, t)}_{\text {drainage solution }}+\varepsilon \underbrace{h(\theta, t)}_{\text {perturbation }}, \quad \varepsilon \ll 1 .
$$

The perturbation $\varepsilon h$ corresponds to the difference between the perturbed film dimensionless thickness $\bar{H}$ and the smooth draining solution $H$. Note that the same lubrication equation (1) and nondimensional parameters have been used by Trinh et al. [5]. However, the decomposition into drainage solution and perturbation is slightly different; in our case, the entire temporal dependence of the perturbation is contained in $h(\theta, t)$. The implications of the different definitions are discussed in Sec. IV A.

The structure of the flow is reported in Fig. 2. The thin film occupies the most part of the domain and connects to a small pool, which forms at the bottom of the cylinder. The Bond number affects the curvature of the meniscus bridging the pool and the film [see inset of Fig. 2(a)]. Increasing the Bond number leads to an increase of the curvature of this meniscus, while no significant changes of the film thickness could be registered at the north pole. In contrast, changing $\delta$ directly affects the volume of fluid in the cylinder relative to its size, thereby impacting all quantities as anticipated by dimensional analysis.

We now proceed to derive the linear perturbation equations of the system. We substitute the decomposition (2) into the nonlinear film thickness evolution (1) and consider first-order terms in $\varepsilon$ only to obtain:

$$
\mathbf{h}_{t}+\mathbf{L}(H, \mathrm{Bo}, \delta) \mathbf{h}=\mathbf{0},
$$

where $\mathbf{L}=\mathbf{L}(H, \mathrm{Bo}, \delta)$ is the linear operator which depends on the drainage solution, the Bond number, and the aspect ratio. The detailed expression of $\mathbf{L}$ is given in Appendix B. 


\section{B. Transient growth analysis}

The drainage solution being space and time dependent, the linear operator in Eq. (3) is a function of the polar angle $\theta$ and is not autonomous. As a consequence, we cannot use a normal mode ansatz for the perturbation and we therefore proceed to a nonmodal analysis. With this method, we seek for the amplification of the perturbation at finite times.

We start by defining the gain function that we will be using to find optimal initial conditions. In our study we consider the ratio of the square of the actual perturbation norm to the initial value. A $L_{2}$ norm is chosen since both variations in potential energy and in surface energy are proportional to $\langle\mathbf{h} \mid \mathbf{h}\rangle$ [22]. Thereby, the gain at the temporal horizon $T$ reads

$$
G(T)=\frac{\langle\mathbf{h}(T) \mid \mathbf{h}(T)\rangle}{\left\langle\mathbf{h}_{0} \mid \mathbf{h}_{0}\right\rangle},
$$

where the Hermitian scalar product for real quantities on the geometry $\Omega=\{\theta \mid \theta \in[0,2 \pi]\}$ is defined by

$$
\langle\mathbf{a} \mid \mathbf{b}\rangle=\int_{\Omega} \mathbf{a}^{T} \mathbf{b} d \theta=\mathbf{a}^{T} \mathbf{M b} .
$$

In order not to favor perturbations at a specific location, a mass matrix with uniform weights is employed:

$$
\mathbf{M}=\frac{2 \pi}{N} \mathbf{I},
$$

with $N$ being the number of discretization points and $\mathbf{I}$ being the identity matrix. The initial condition is given by $\mathbf{h}_{0}=\mathbf{h}(0)$.

For time-independent operators, the perturbation's evolution can be computed using the propagator operator. The optimal initial condition is directly obtained by the singular value decomposition of the propagator operator [23]. While such eigenvalue methods can also be generalized for time-dependent operators [24,25], the optimal growth can also be conveniently obtained with a variational formulation and adjoints fields [16]. A comparison between the two methods has been pursued by Mao et al. [25], showing that eigenvalue approaches are preferable when dealing with large-dimensional problems.

Here, the constraints resulting from the governing equation and the initial condition are enforced via the Lagrange multipliers $\mathbf{h}^{\dagger}$ and $\mathbf{h}_{0}^{\dagger}$, respectively. Since these constrains have to be fulfilled locally, the Lagrange multipliers are space (and time, for $\mathbf{h}^{\dagger}$ ) dependent. They are governed by the so-called adjoint equations. The augmented Lagrangian consisting of the objective function to be optimized and the constraints reads

$$
\mathcal{L}\left(\mathbf{h}, \mathbf{h}^{\dagger}, \mathbf{h}_{0}, \mathbf{h}_{0}^{\dagger} ; T\right)=\underbrace{G(T)}_{\text {gain }}-\underbrace{\int_{0}^{T}\left\langle\mathbf{h}^{\dagger} \mid \mathbf{h}_{t}+\mathbf{L h}\right\rangle d t}_{\text {enforcing governing equation }}-\underbrace{\left\langle\mathbf{h}_{0}^{\dagger} \mid \mathbf{h}(0)-\mathbf{h}_{0}\right\rangle}_{\text {enforcing initial condition }} .
$$

The last two terms of Eq. (7) penalize the Lagrangian if the fields $\mathbf{h}$ and $\mathbf{h}_{0}$ do not satisfy the governing equation (3) and the initial condition.

The adjoint fields equations are obtained by setting to zero the first variations of $\mathcal{L}$ with respect to any variation $\delta \mathbf{h}$ (see derivation in Appendix C):

$$
\begin{aligned}
\left\langle\frac{\partial \mathcal{L}}{\partial \mathbf{h}} \mid \delta \mathbf{h}\right\rangle=0, \quad \forall \delta \mathbf{h} \Leftrightarrow & \mathbf{h}^{\dagger}(T)=2 \frac{\mathbf{h}(T)}{\left\langle\mathbf{h}_{0} \mid \mathbf{h}_{0}\right\rangle}, \\
& \mathbf{h}_{t}^{\dagger}-\mathbf{L}^{\dagger} \mathbf{h}^{\dagger}=\mathbf{0} \quad t \in[0, T], \\
& \mathbf{h}_{0}^{\dagger}=\mathbf{h}^{\dagger}(0)
\end{aligned}
$$




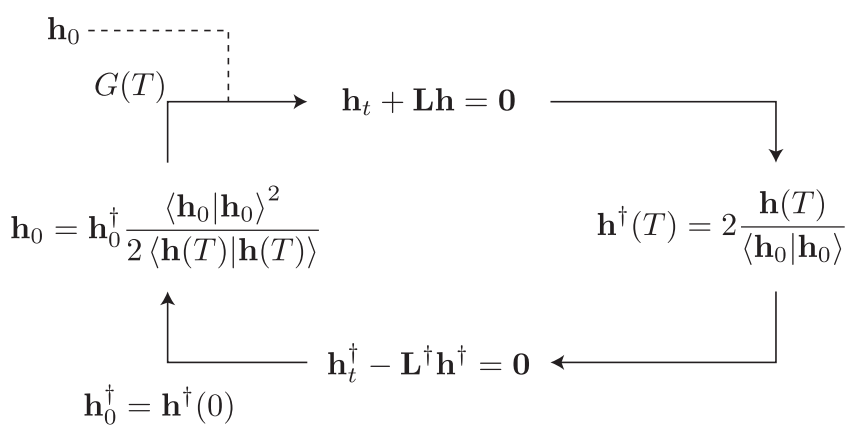

FIG. 3. Sketch of the iterative procedure to find the optimal gain and initial condition for a given time horizon $T$, Bond number Bo, and film aspect ratio $\delta$.

where $\mathbf{L}^{\dagger}$ is the adjoint operator, defined as $\langle\mathbf{a} \mid \mathbf{L b}\rangle=\left\langle\mathbf{L}^{\dagger} \mathbf{a} \mid \mathbf{b}\right\rangle$. Instead of analytically finding the continuous adjoint of the direct operator, we follow Schmid and Henningson [23] and use the discrete adjoint, which for an identity weight matrix, see Eq. (6), reduces to the transpose: $\mathbf{L}^{\dagger}=\mathbf{L}^{T}$. The adjoint equation (9) has to be integrated backward in time with the terminal condition given by Eq. (8). The Lagrange multiplier $\mathbf{h}_{0}^{\dagger}$ turns out to be the final value of the adjoint field $\mathbf{h}^{\dagger}$. Additionally, the stationarity condition with respect to the control variable $\mathbf{h}_{0}$ yields the optimality condition:

$$
\left\langle\frac{\partial \mathcal{L}}{\partial \mathbf{h}_{0}} \mid \delta \mathbf{h}_{0}\right\rangle=0, \quad \forall \delta \mathbf{h}_{0} \Leftrightarrow \mathbf{h}_{0}=\mathbf{h}_{0}^{\dagger} \frac{\left\langle\mathbf{h}_{0} \mid \mathbf{h}_{0}\right\rangle^{2}}{2\langle\mathbf{h}(T) \mid \mathbf{h}(T)\rangle} .
$$

By deriving the augmented Lagrangian by their multipliers one retrieves the constraints.

In order to compute the optimal initial conditions, the algorithmic approach schematized in Fig. 3 is employed. The linear equation Eq. (3) is integrated forward in time from $t=0$ to the chosen time horizon $t=T$, using an arbitrary initial condition $\mathbf{h}_{0}$. The initial condition for the adjoint equation is then computed from Eq. (8) and the adjoint equation (9) is integrated backward in time from $t=T$ to $t=0$. Using Eq. (10), the new guess of the optimal initial condition is then given by Eq. (11). The forward and backward integrations have to be continued until convergence of the gain $G(T)$ introduced in Eq. (4). Since the direct and adjoint linear operators are not mass preserving, the mass of the initial condition is subtracted at every iteration to find zero-mass optimal perturbations. If this step is omitted, the optimality condition would be biased by initial perturbations adding mass to the system. Note that this constraint could also have been included as a constraint in the augmented Lagrangian (7). However, for the imposition of such a single scalar constraint, the use of a projection method is equivalent from a computational-cost point of view. At first order, the zero-mass condition for the perturbations is

$$
m=\int_{\Omega} h_{0}(\theta)\left[\frac{1}{\delta}-H(\theta, 0)\right] d \theta=\left(\frac{1}{\delta}-1\right) \int_{\Omega} h_{0}(\theta) d \theta=0,
$$

where the uniform initial drainage profile $H(\theta, 0)=1$ has been used. The resulting gain $G(T)$ and the initial condition $\mathbf{h}_{0}$ are the optimal for the time horizon $T$. By repeating the algorithm for several time horizons, all other things being equal, one obtains the envelope of the optimal amplifications for a given Bond number and film aspect ratio $\delta$. For each increment of horizon time $T$, the optimal initial condition of the previous time horizon is used as the initial guess. Examples of such quantities are given in Fig. 4 and are discussed in Sec. III A.

The final step of our method consist in identifying the maximum $G_{\max }$ of $G(T)$ for each couple $(\mathrm{Bo}, \delta)$, which is associated to an optimal growth and initial condition. Examples are shown in Fig. 6 and are discussed in Secs. III B and III C. Prior to commenting on the obtained results, we briefly evoke our numerical methods. 


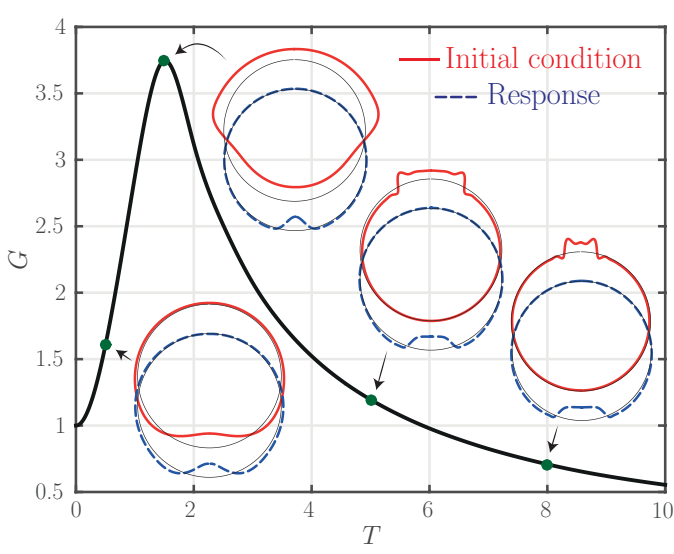

(a)

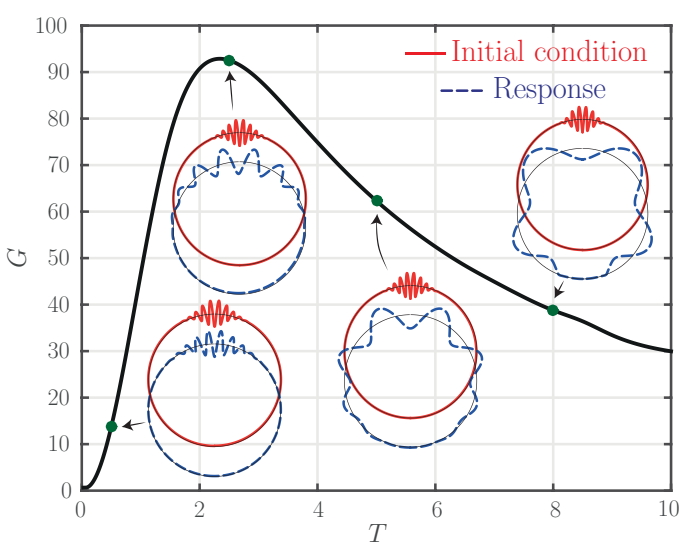

(b)

FIG. 4. Optimal gains as a function of the time horizon for two Bond numbers: (a) Bo $=10$ and (b) Bo $=50 . \delta=10^{-2}$. For visualization purposes the initial conditions (solid red) and responses (dashed blue) are normalized by their respective largest value and magnified by 20 .

\section{Numerical methods}

A numerical procedure was developed to solve the highly nonlinear Eq. (1) as well for the transient growth study. The polar angle $\theta \in[0,2 \pi]$ is uniformly discretized with $N$ collocation points. We exploit the periodicity of the domain and use Fourier spectral methods [26] to compute spatial derivatives with a high degree of accuracy [27]. The effect of numerical diffusion is minimized by performing the time integration with the second-order Crank-Nicolson MATLAB routine ode23t.m.

It is important to state that the discretization scheme is accurate enough to avoid the amplification of numerical errors up to $\mathrm{Bo} \approx 350$. The base flow solution is therefore exempt of transient waves and can be used for the stability analysis for the considered Bond number range.

First, the drainage solution for a given set of physical and geometrical parameters is calculated with a computation time of the order of a few minutes on a single Intel core at $2.6 \mathrm{GHz}$. Second, the iterative scheme giving the optimal condition is ran. In order to reduce the memory-storage requirement, the direct and adjoint linear operators are built at several time steps and not computed a priori for the entire time interval. Yet, due to the repeated forward and backward integrations, the computation of the optimality conditions and the evaluation of the largest gain are computationally more expensive than the first step. The computation of the optimal conditions for the entire Bond number and aspect ratios range is of the order of few days, depending on the number of time horizons considered. For more details and a series of validation tests see Appendix D.

To complement these tools we used the open source two-phase 2D-Stokes solver Ulambator [28] (see Sec. IV B).

\section{RESULTS}

\section{A. Optimal perturbation regimes}

The results of the optimal transient growth analysis are presented hereafter; a deeper discussion follows in Sec. IV. The dependence of the optimal gains as a function of the time horizon, as well as some corresponding initial perturbations and resulting responses, are presented on Fig. 4 for two different Bond numbers, $\mathrm{Bo}=10$ and $\mathrm{Bo}=50$. Common to all explored cases is the shape of $G(T)$, where the gain tends to zero for large enough values of $T$, revealing the asymptotic stability of the film. As a consequence, we focus our attention on the amplifications over a short, yet finite, time period.

Although the system is asymptotically stable independently of the parameters, the respective values of $G_{\max }$ depend significantly on the Bond number, ranging from less than 4 for Bo $=10$ in 


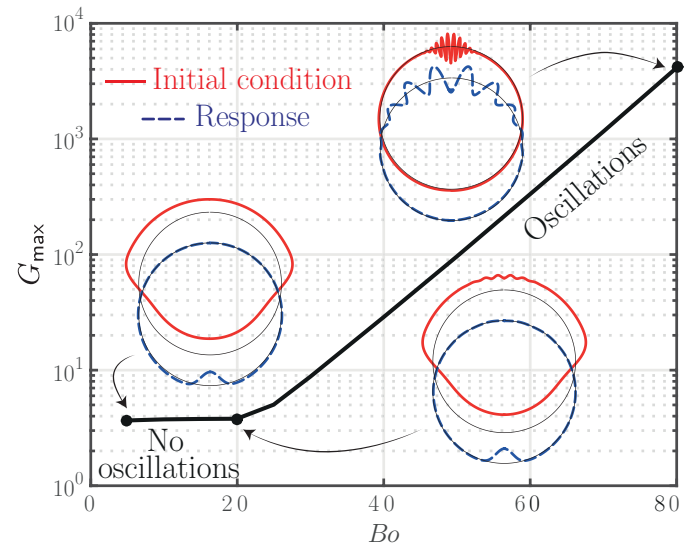

(a)

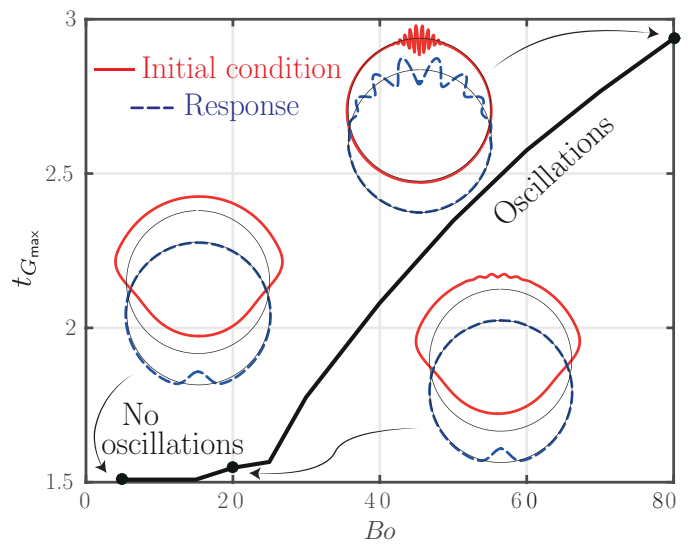

(b)

FIG. 5. Largest optimal gains $G_{\max }$ (a) and time horizons $t_{G_{\max }}$ (b) as a function of the Bond number. $\delta=10^{-2}$. For visualization purposes the initial conditions (solid red) and responses (dashed blue) are normalized by their respective largest value and magnified by 20 . The two regimes are highlighted.

Fig. 4(a) to more than 90 for Bo $=50$ in Fig. 4(b). In addition to this quantitative difference, the two cases also differ qualitatively. The optimal initial conditions for $\mathrm{Bo}=10$ correspond to perturbations with a front whose location gets closer to the north pole as the time horizon increases. In fact, these fronts are solely convected downstream by the draining flow until the final time $T$. Their maximal gain is achieved when the front is close to the equator, here for $T \approx 1.5$. On the other hand, for Bo $=50$, the optimal initial conditions are oscillations of the interface at the top of the geometry. They are largely amplified (not visible on the responses of Fig. 4 because of normalization) and travel downstream.

\section{B. Influence of the Bond number}

When increasing the Bond number for a given film aspect ratio $\delta$, the optimal perturbation switches from a front to oscillations at the top as shown in Fig. 5(a) along with the values of the largest obtainable gain [and the corresponding optimal time in Fig. 5(b)]. The transition between these two type of instabilities is identified. For $\delta=10^{-2}$, the critical Bond number corresponding to the transition from nonoscillating to oscillating perturbations is around 20. Nonoscillating perturbations display a constant maximal optimal gain $G_{\max }$ which is reached at the same time horizon of $T \approx 1.5$. At $\mathrm{Bo}=20$, the optimal initial condition corresponds to a mix of front and oscillating perturbations and is obtained at a slightly later time. The draining front is still present, but oscillations start to appear at the north pole. For higher Bond numbers, the oscillating perturbations are characterized by a largest optimal gain whose value increases exponentially with the Bond number. Although the draining flow makes the system asymptotically stable, the potential amplification due to the Rayleigh-Taylor instability at short times is unbounded as the Bond number increases. The system behaves as a (very effective) noise amplifier. The corresponding optimal time as a function of Bo is found to follow a power law with exponent of $\sim 0.2$. Broadly speaking, as the Bond number increases, the Rayleigh-Taylor instability has more time to grow to larger gains before being dampened by the thinning of the film.

\section{Influence of the film aspect ratio}

The problem's aspect ratio, $\delta$, impacts the low Bond number regime (see Fig. 6 ). The gains of the frontlike perturbations are larger as the film aspect ratio decreases, and those values are attained at slightly larger times. Similarly, $\delta$ has an effect on the transition between the two type of instabilities. 


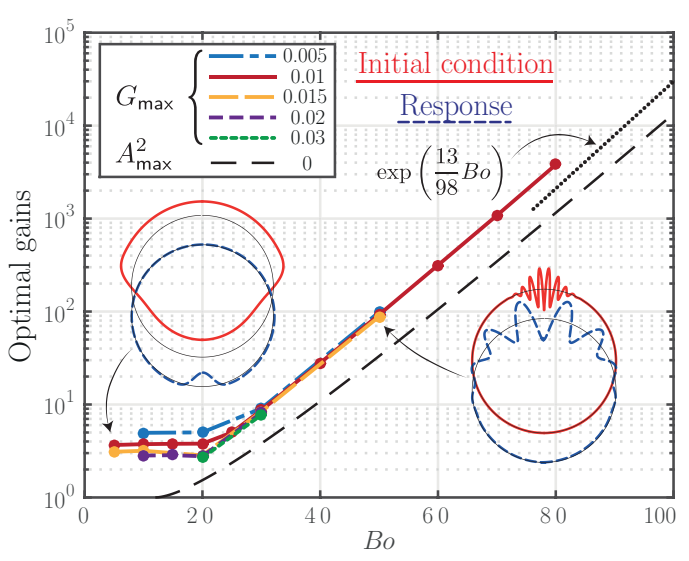

(a)

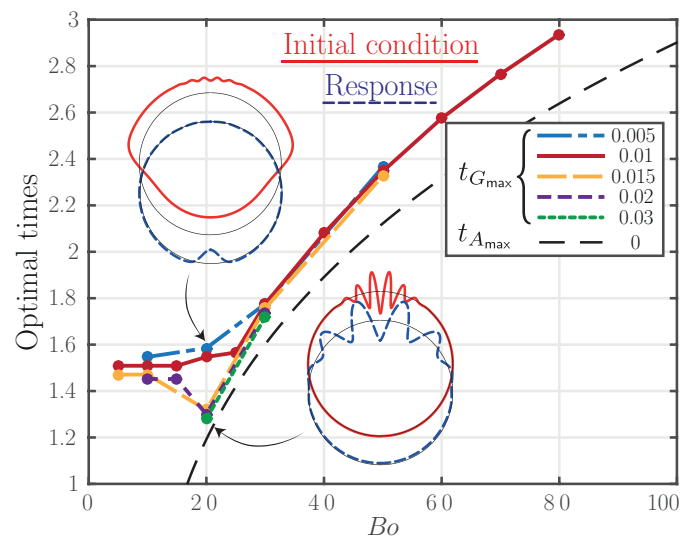

(b)

FIG. 6. Largest optimal gains $G_{\max }$ (a) and time horizons $t_{G_{\max }}$ (b) as a function of the Bond number for several film aspect ratios $\delta$ (see legend). For visualization purposes the initial conditions (solid red) and responses (dashed blue) are normalized by their respective largest value and magnified by 20. Long dashed black lines correspond to the square of the optimal amplifications at the top, $A_{\max }^{2}$, and the corresponding optimal times, $t_{A_{\max }}$; see Sec. IV A. Black dotted line in panel (a) corresponds to the optimal gain evolution limit for large Bond numbers; see Sec. IV A.

In Fig. 6(b) one observes that for $\mathrm{Bo}=20$ a thin film gives rise to a front perturbation, whereas for a thick film, the perturbation is oscillatory. In contrast, $\delta$ has very little effect on the high Bond number regime. This means that the geometrical parameters of the problem are solely captured by the Bond number defined by the combination of $R$ and $H_{i}^{*}$. This fact is confirmed by the collapse of curves in Figs. 6(a) and 6(b). In particular, this master curve displays the earlier evoked exponential dependance of $G_{\max }$ on the Bond number [Fig. 6(a)], which is discussed next.

\section{DISCUSSION}

\section{A. Optimal transient growth analysis at $\boldsymbol{\theta}=\mathbf{0}$}

The perturbations giving rise to significant optimal gains $G_{\max }$ have been found to be oscillations of the film interface around the north pole. It is therefore natural to consider the top region, in agreement with the work of Trinh et al. [5]. In the following, we will use and extend their linear stability analysis to rationalize our results.

If one focuses only on the region at the north pole $\left(\theta=\delta^{1 / 2} x\right.$ for $\left.\delta \rightarrow 0\right)$, the lubrication equation (1) can be greatly simplified in the asymptotic limit of small aspect ratios. For an initial harmonic perturbation of wave number $k, h(x, 1)=\exp (i k x)$, the resulting linear perturbation equation has the following analytical solution:

$$
h(x, \tilde{t})=A(k, \tilde{t}) \exp \left[i\left(k \frac{x}{\tilde{t}^{3 / 2}}\right)\right],
$$

with the amplitude being a function of the wave number $k$ and the rescaled time $\tilde{t}=1+2 t / 3$ :

$$
A(k, \tilde{t})=\underbrace{\frac{1}{\tilde{t}^{3 / 2}}}_{\mathrm{I}} \underbrace{\exp [\underbrace{\left(1-\tilde{t}^{-7 / 2}\right) \frac{k^{2}}{7}}_{\mathrm{a}}-\underbrace{\frac{1}{\mathrm{Bo}}\left(1-\tilde{t}^{-13 / 2}\right) \frac{k^{4}}{13}}_{\mathrm{b}}]}_{\mathrm{II}} .
$$




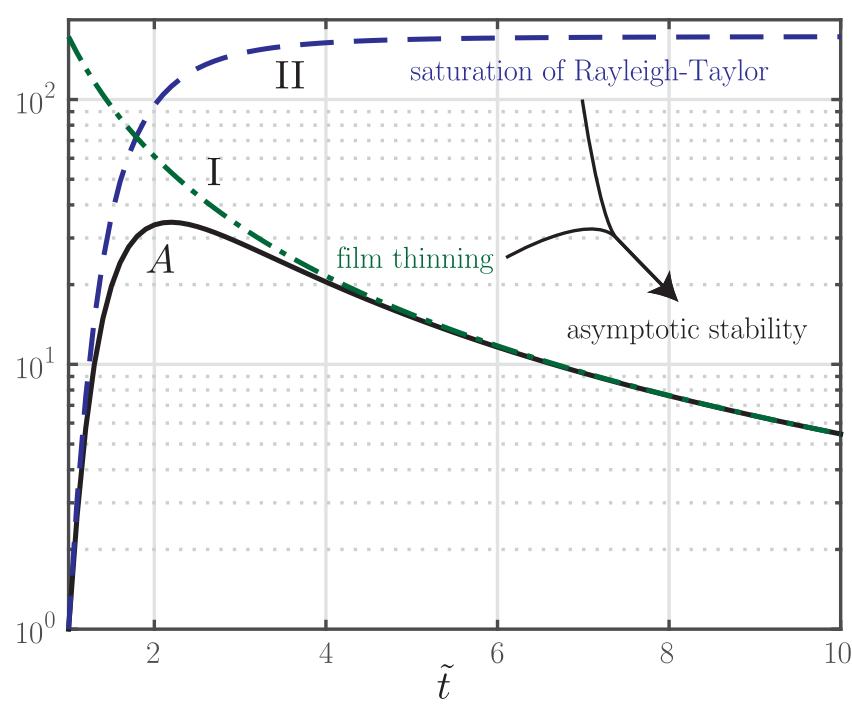

FIG. 7. Temporal evolution of the gain at $\theta=0$ given by Eq. (14), $A$, for Bo $=100$ and $k=7$ (solid line), Rayleigh-Taylor exponential contribution II (dashed line), and pure film-thinning contribution I (dash-dotted line) of Eq. (14). Term I is rescaled by the final value of II, namely $\exp (13 \mathrm{Bo} / 196)$; see text. Note that $A$ is the product of terms I and II.

In this case, the amplitude squared $A(k, \tilde{t})^{2}$ corresponds to our definition of the perturbation's gain [see Eq. (4)] for $\theta=0$.

The $-3 / 2$ power-law dependence of the amplitude on the rescaled time in term I differs from the value of -1 obtained by Trinh et al. [5]. The reason is the different film thickness decompositions. We consider $\bar{H}(x, \tilde{t})=H(\tilde{t})+\varepsilon h(x, \tilde{t})$, whereas in Ref. [5] the decomposition is $\bar{H}(x, \tilde{t})=H(\tilde{t})[1+$ $\varepsilon \eta(x, \tilde{t})]$. Since $H(\tilde{t})$ decreases monotonically in time at $\theta=0$ [see Fig. 1(b)], the perturbation $h(x, \tilde{t})=H(\tilde{t}) \eta(x, \tilde{t})$ increases in time only if the growth of $\eta(x, \tilde{t})$ overcomes the decay in $H(\tilde{t})$; a growth in $\eta(x, \tilde{t})$ does not imply a growth in $h(x, \tilde{t})$. Trinh et al. [5] find that $\eta(x, \tilde{t})$ grows for Bond numbers larger than 8 . With our definition, the necessary value for initial growth of $h(x, \tilde{t})$ becomes 12 .

By definition, the dimensionless drainage time is of order 1 for all Bond numbers. On the contrary, the time scale associated with the growth of the Rayleigh-Taylor instability varies with Bo; it is typically of the order of 1/Bo [11]. The difference in the time scales may lead to transient growths for sufficiently large Bond numbers. The threshold may be obtained comparing the initial temporal dependence of the contributions I and II in Eq. (14). At first order for $\tilde{t} \rightarrow 1$, the thinning term I evolves as $1-3(\tilde{t}-1) / 2$, whereas the largest value of the Rayleigh-Taylor term II is found for $k=\sqrt{\mathrm{Bo} / 2}$ and increases as $1+\mathrm{Bo}(\tilde{t}-1) / 8$. Initial growth of perturbations at the top only occurs if the instability grows faster than the thinning of the film, which is indeed the case for Bo $>12$.

The analytical expression (14) is now employed to elucidate the reasons of the algebraic growth at finite transient times. The evolution of the gain $A$ as a function of the rescaled time $\tilde{t}$ is shown in Fig. 7 for $\mathrm{Bo}=100$. At short times, an exponential growth of the gravitational instability is observed (as Bo > 12). The Rayleigh-Taylor contribution II of Eq. (14) dominates the flow; the thinning term I is close to unity. Term II incorporates the destabilizing and stabilizing components of the Rayleigh-Taylor instability, namely IIa and IIb. As a result of the thinning of the film with time, the instability saturates to a plateau (see curve II on Fig. 7), thereby increasing the relative importance of part I which scales as $\tilde{t}^{-3 / 2}$ (see curve I on Fig. 7). This stabilizing effect therefore dominates the saturated exponential growth of the instability at large times (see curve $A$ on Fig. 7), so that only a transient growth scenario may effectively destabilize the fluid's interface. 


\section{RAYLEIGH-TAYLOR INSTABILITY UNDER CURVED ...}

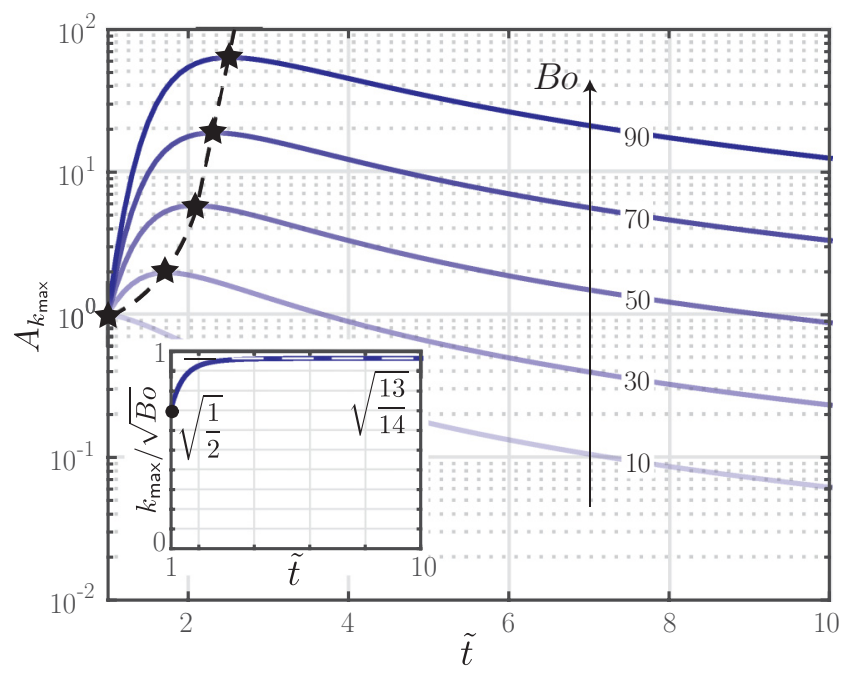

FIG. 8. Gain $A_{k_{\max }}=A\left(k_{\max }, \tilde{t}\right)$ as a function of time for different Bond numbers. Inset: normalized optimal wave number $k_{\max } / \sqrt{\mathrm{Bo}}$. Stars and dashed line indicate the largest transient growth and the corresponding times. Full circle (dashed line) in the inset corresponds to the short (long) time limit of the normalized optimal wave number.

The analytical solution (14) for the gain $A$ is now optimized in the wave-number-time space to find the optimal transient growth. The optimization procedure reduces to finding a maximum of the two arguments function $A(k, \tilde{t})$ (the Bond number is here a parameter). The optimal wave number is obtained by solving $\partial A / \partial k=0$ :

$$
k_{\max }(\tilde{t})=\sqrt{\frac{13}{14} \frac{\left(1-\tilde{t}^{-7 / 2}\right)}{\left(1-\tilde{t}^{-13 / 2}\right)} \text { Bo. }}
$$

In the short time limit, $\tilde{t} \rightarrow 1$, we recover $k_{\max }=\sqrt{\mathrm{Bo} / 2}$ classically obtained for the Rayleigh-Taylor instability under a horizontal plane and in Ref. [5]. For large optimal times, $\tilde{t} \gg 1, k_{\max }$ converges to $\sqrt{13 \mathrm{Bo} / 14}$, so that the relative change in the optimal wave number is weak. Note that $k_{\max }(\tilde{t})$ is not the evolution of the perturbation wave number, but is the initial wave number giving rise to the largest amplitude $A_{k_{\max }}$ at time $\tilde{t} . A_{k_{\max }}$ can be found by substituting the expression of $k_{\max }(\tilde{t})$ in Eq. (14):

$$
A_{k_{\max }}(\tilde{t})=\frac{1}{\tilde{t}^{3 / 2}} \exp \left[\frac{13}{196} \frac{\left(1-\tilde{t}^{-7 / 2}\right)^{2}}{\left(1-\tilde{t}^{-13 / 2}\right)} \text { Bo }\right] .
$$

The optimal wave number as well as the resulting gain obtained for different Bond numbers are presented in Fig. 8. The fast convergence of $k_{\max }$ to the large time limit $\sqrt{13 \mathrm{Bo} / 14}$ is shown in the inset.

The optimal times and gains, highlighted by stars and the dashed line in Fig. 8, are found by solving $\partial A_{k_{\max }} / \partial \tilde{t}=0$. No analytical expression was found and a numerical resolution had to be performed. The optimal times and the corresponding optimal gains at the top are reported in Fig. 6 .

We now compare these results of the optimization of the transient growth at $\theta=0$ for $\delta \rightarrow 0$ to the ones presented in Sec. III for the optimization over all angles and finite aspect ratios. The optimal gain $A_{\max }^{2}$ obtained semianalytically at the top follows the same exponential dependence in Bond number as the one of $G_{\max }$ earlier obtained by the optimal transient growth analysis on the entire geometry [see Fig. 6(a)]. The difference in their exact values is imputable to a difference in the norm used to evaluate each case. $A_{\max }^{2}$ accounts only for the perturbation value at $\theta=0$ by contrast with $G_{\max }$ which covers the entire domain [see Eq. (4)]. 


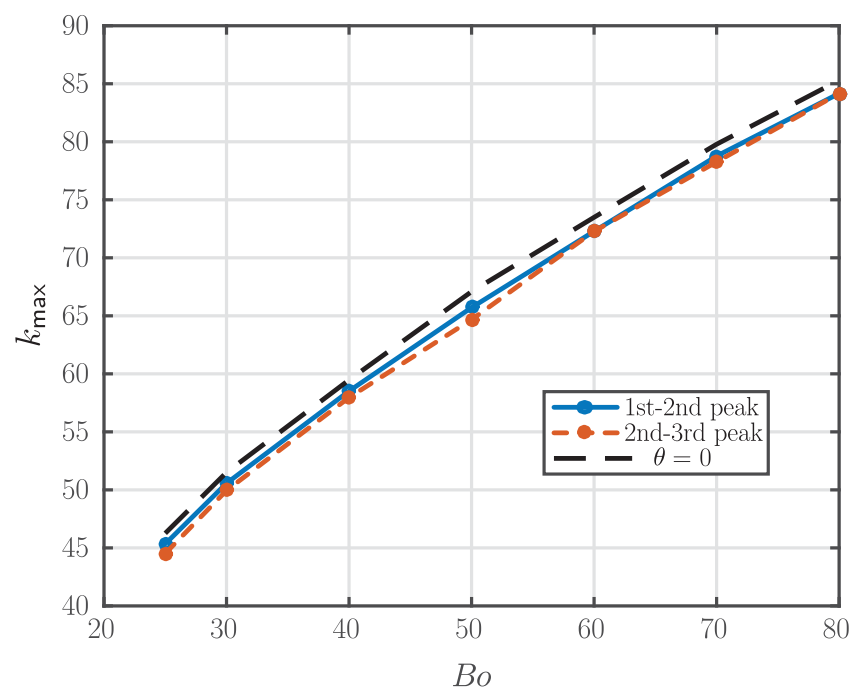

FIG. 9. Wave numbers corresponding to the optimal initial condition obtained from the transient growth analysis on the entire geometry for $\delta=10^{-2}$ (solid and dotted lines) and at the top, Eq. (15) multiplied by $\delta^{-1 / 2}$ to convert from $x$ to $\theta$ space (dashed line), with $\tilde{t}$ maximizing $A_{k_{\max }}$. The $k_{\max }$ of the transient growth analysis over all $\theta$ are the wave numbers corresponding to the distance between the first and second or second and third peaks of the oscillations, respectively.

We note that the variation of the algebraic growth with the Bond number tends to exp(13Bo/98) for $\mathrm{Bo} \gg 1$. This is intrinsically related to the wave-number stretching effect. In fact, the drainage stretches the perturbations as the effective wave number in Eq. (13) is $k / \tilde{t}^{3 / 2}$ [see the plots in Fig. 4(b) for an illustration]. As a result, the stabilizing term IIb of Eq. (14) evolves faster than its destabilizing counterpart, IIa. If the perturbations were not stretched, i.e., if the destabilizing and stabilizing terms IIa and IIb of Eq. (14) would have the same temporal dependence, the exponential increase of the gain would tend to $\exp (\mathrm{Bo} / 2)$; the algebraic growth would solely be dictated by the thinning contribution of the draining flow. The mismatch between the two components of term II adds to this effect, reducing the prefactor from $1 / 2$ to $13 / 98$. In the context of viscous filaments, the wave-number stretching has also been shown to be stabilizing [29].

Regarding the optimal times shown in Fig. 6(b), both $t_{G_{\max }}$ and $t_{A_{\max }}$ follow a similar power law with an exponent smaller than unity, typically $\sim 0.2$. The shape of the optimal perturbations can be compared as well. In both cases, the optimal perturbations are oscillations of the interface close to the top. This is a result for the optimal transient growth analysis on the entire circle and an assumption for the analysis at the pole. However, for the analysis over all $\theta$, their wave number and amplitude decrease in space as the distance from $\theta=0$ increases [see Fig. 4(b), for example]. Thus, only the first and second apparent wave numbers, which are close to the pole, will be considered for the comparison. A very good correspondence of the wave numbers of the optimal initial conditions as obtained in the two approaches is shown on Fig. 9. In conclusion, the optimal perturbations found by the analysis at the top, slightly modulated in space, are optimal for the problem on the entire geometry for Bo $\gtrsim 20$. Note that the analysis at $\theta=0$ holds in the limit of a vanishing film aspect ratio, which turns out to be a valid assumption since the oscillating regime has been found not to directly depend on $\delta$ (see Sec. III C).

\section{B. Transition to dripping droplets}

The initial growth analysis at the top tells us that below Bo $=12$ no droplets can be formed, whereas from the transient growth analysis on the entire geometry we know that below Bo $\approx 20$ the 


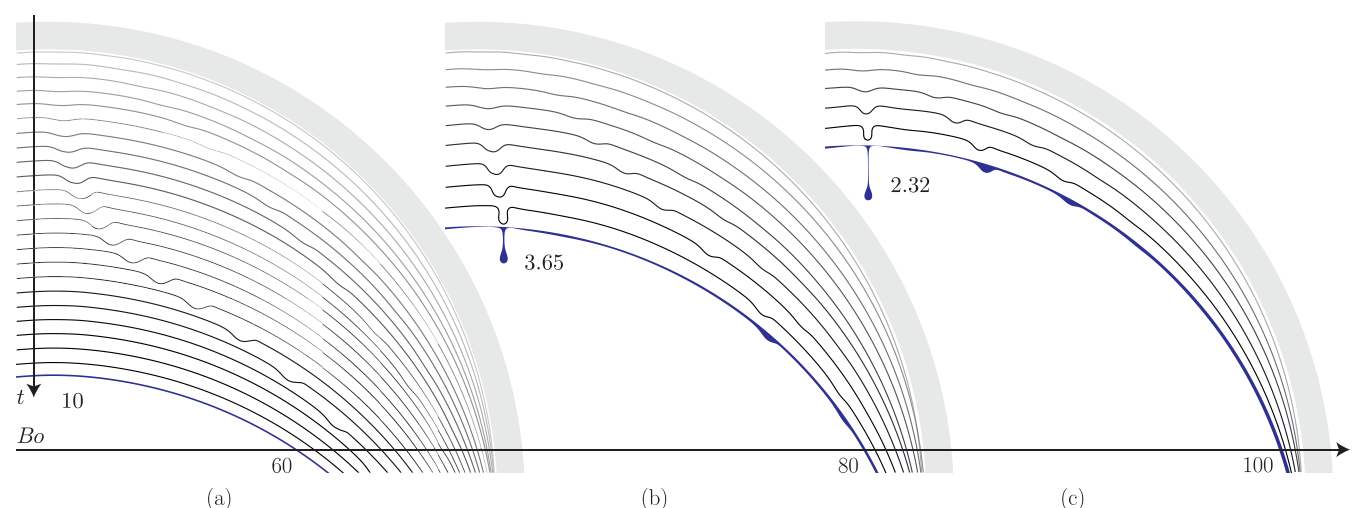

FIG. 10. Nonlinear evolution of the interface initially perturbed by the optimal initial condition $h_{0}^{\max }$ obtained by the linear stability for the specific Bond number. The initial condition reads $\bar{H}(\theta, 0)=$ $1+a h_{0}^{\max } /\left\|h_{0}^{\max }\right\|_{\infty}$ with $a=2.25 \times 10^{-2}$. The film aspect ratio is $\delta=10^{-2}$ and the Bond numbers are (a) $\mathrm{Bo}=60$, (b) Bo $=80$, and (c) Bo $=100$. Gray lines correspond to the interface shapes at successive time instants; the time step is 0.4 . The filled profiles correspond to the liquid films at the indicated final time when the droplet is reabsorbed (a) or when pinching off occurs (b), (c). No dripping occurs even at larger times for the case (a).

optimal perturbations do not correspond to oscillations of the film at the top. We classify droplets in two categories: Transient droplets grow for a certain time before being reabsorbed by the film whereas dripping droplets pinch off from the substrate (see Figs. 10 and 11). We now turn to the question of dripping droplets.

In view of the exponentially increasing algebraic gain with the Bond number in the oscillating regime, we argue that the system acts as an effective amplifier. Initial perturbations are crucial. They project onto the most unstable perturbation which will emerge in the growth at finite times. The linear evolution initially dictates the growth of the perturbations, but as they reach a certain amplitude, nonlinear effects enter in consideration (see Fig. 12). In particular, the linear dynamics

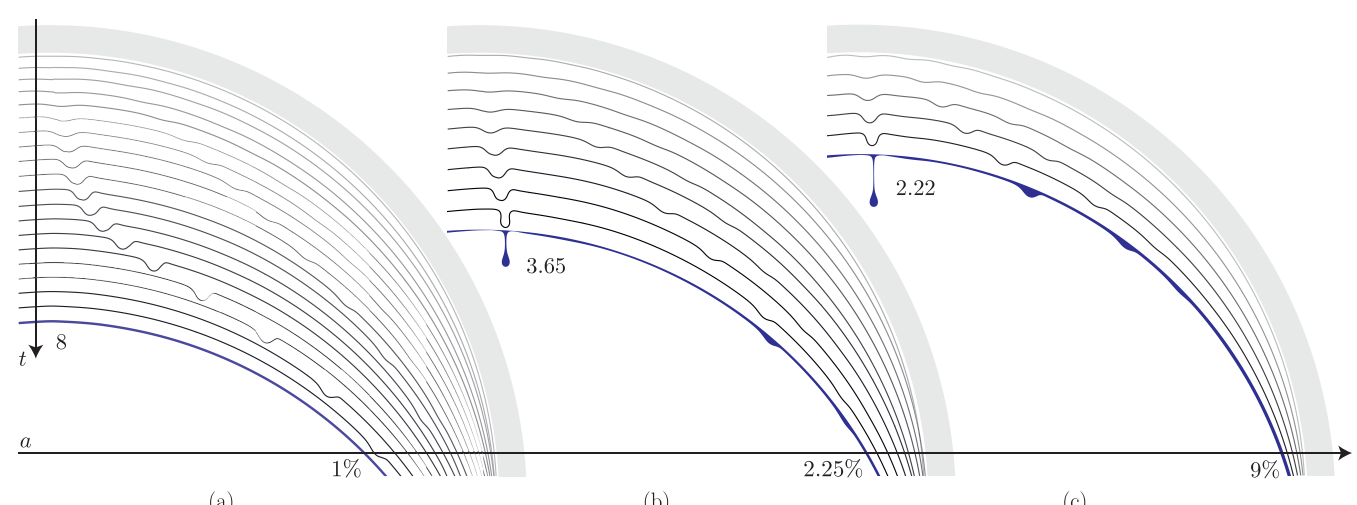

(a)

(b)

(c)

FIG. 11. Nonlinear evolution of the interface initially perturbed by the optimal initial condition $h_{0}^{\max }$ obtained by the linear stability for Bo $=80$. The initial condition reads $\bar{H}(\theta, 0)=1+a h_{0}^{\max } /\left\|h_{0}^{\max }\right\|_{\infty}$ with (a) $a=10^{-2}$, (b) $a=2.25 \times 10^{-2}$, and (c) $a=9 \times 10^{-2}$. The film aspect ratio is $\delta=10^{-2}$. Gray lines correspond to the interface shapes at successive time instants; the time step is 0.4 . The filled profiles correspond to the liquid films at the indicated final time when the droplet is reabsorbed (a) or when pinching off occurs (b), (c). No dripping occurs even at larger times for the case (a). 


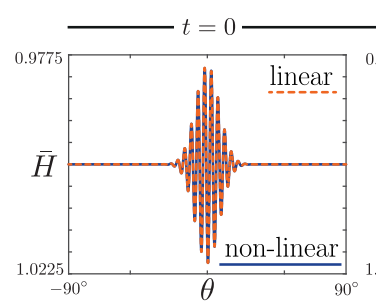

(a)

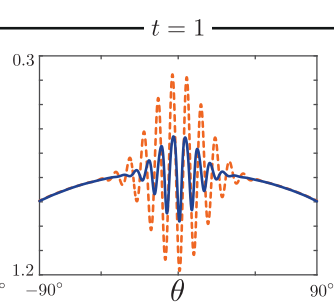

(b)

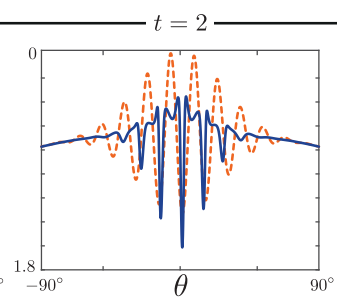

(c)

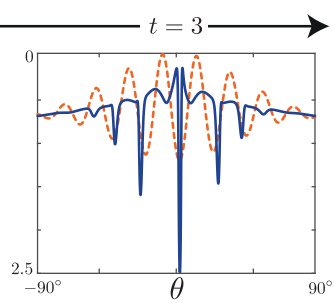

(d)

FIG. 12. Linear (dashed) and nonlinear (solid) evolutions of the interface initially perturbed by the optimal initial condition $h_{0}^{\max }$ obtained by the linear stability for the specific Bond number. The initial condition reads $\bar{H}(\theta, 0)=1+a h_{0}^{\max } /\left\|h_{0}^{\max }\right\|_{\infty}$ with $a=2.25 \times 10^{-2}$. The film aspect ratio is $\delta=10^{-2}$ and the Bond number is $\mathrm{Bo}=80$. Dripping will occur at $t=3.65$. The linear evolution is obtained by the resolution of the linearized lubrication equation (3) for the disturbances $h$ on a draining flow $H$ given by Eq. (1), whereas the nonlinear evolution is obtained by the resolution of the two-phase 2D Stokes equations. Note that the scale of the $y$ axis changes.

initially presents a faster increase of the disturbance amplitudes as well as a slightly larger sliding velocity of the droplets [see Fig. 12(b)]. The peculiar shape of the front and rear menisci of the sliding droplets on a thin wetting precursor film [see Figs. 12(c) and 12(d)], and the eventual pinch off, are purely nonlinear effects [30].

We expect that the transition from transient droplets to dripping droplets is dependent on the initial perturbation intensity. For large initial perturbations, the necessary amplification in order to trigger the formation of droplets is smaller; i.e., the transition to dripping droplets will occur already for smaller Bond numbers. On the other hand, when the film is only weakly disturbed, a larger Bond number is necessary to create dripping droplets before drainage makes the film too thin. Given a perturbation amplitude, there exists therefore a critical Bo above which the linear growth results in the formation of droplets which pinch off. Conversely, given a Bond number, there likely exists a critical initial disturbance amplitude for the transition to dripping droplets.

To support our argument, we performed nonlinear numerical simulations using the two-phase 2D-Stokes solver Ulambator [28] for different Bond numbers and perturbation amplitudes. The resulting film profiles for $\mathrm{Bo}=60,80$, and 100 , perturbed at $t=0$ by the optimal initial condition found by the linear analysis at the given Bond number, with an amplitude of $2.25 \%$ the average initial thickness are shown in Fig. 10. Different sorts of droplets form, depending on the value of the Bond number. In all three cases, droplets are created close to the north pole. For Bo $=60$, after an initial growth, they decay and are reabsorbed by the liquid film, thereby behaving as transient droplets. For $\mathrm{Bo}=80$ and 100, the linear amplification is strong enough for the nonlinear effects to lead to pinch off (keeping in mind the limitation of the two-dimensional geometry). We furthermore investigated the effect of the initial disturbance amplitude on the transition. The nonlinear evolutions for $\mathrm{Bo}=80$ and optimal initial conditions with amplitudes $a=1 \%, 2.25 \%$, and $9 \%$ are shown in Fig. 11. The linear gain for $\mathrm{Bo}=80$ is not large enough to yield dripping droplets when the perturbation amplitude is reduced to $1 \%$ the average initial thickness. We verified that for $\mathrm{Bo}=100$ an amplitude of $1 \%$ is sufficient for dripping droplets to form whereas for Bo $=60$ an amplitude of $9 \%$ is not sufficient for droplets to pinch off. Increasing the Bond number or the initial disturbance amplitude have therefore similar effects. In addition, as the Bond number (or initial amplitude) is larger, the pinch off does occur earlier. Note that for illustration purposes only a fourth of the circle is shown in Figs. 10 and 11; on the rest of the geometry transient droplets occur, but they do not detach from the substrate, and the perturbations are not symmetric.

We now briefly comment on the limitations of our method. First and foremost, our calculation is $2 \mathrm{D}$ although droplet pinch off is a 3D mechanism. We therefore anticipate that the precise value of the critical dripping Bond number will differ if using 3D computations in this section. Nevertheless, we 
anticipate that a similar scenario will occur. Similarly we note that the present theoretical approach accounts for an initial perturbation only. We speculate that a coupling between the microscopic scale and the macroscopic scale similar to the one described by Bertozzi and Brenner [17] is at the origin of the film perturbations necessary for the droplet formation. The sensitivity of the system to perturbations persisting in time goes beyond the aim of this work and is the subject of a separate study.

\section{CONCLUSIONS}

In this study, the Rayleigh-Taylor instability of a thin viscous film coating the interior of a cylindrical substrate has been investigated by performing a linear optimal transient growth analysis. For such a geometry, gravity has a twofold effect. On the one hand, it creates a draining flow that stabilizes the interface. On the other hand the gravitational field destabilizes the free surface and is at the origin of dripping droplets. The exponential growth of perturbations dominates at early times, saturates, and is stabilized by the algebraic dampening of the draining flow at larger times. In the asymptotic limit of large times, the liquid interface is stable with respect to infinitesimal perturbations; and this is for all Bond numbers and all initial film thickness to cylinder radius ratios, $\delta$. Nonetheless, it has been found that droplets may drip from the cylindrical substrate [5]. We therefore performed an optimal transient growth analysis considering the entire geometry at finite times.

By analyzing the influence of the Bond number on the optimal conditions, we have been able to distinguish between two types of optimal perturbations. For surface-tension-dominated flows, the optimal gains are independent of the Bond number and increase for a decreasing film aspect ratio. The corresponding initial conditions display a front, which is solely drained downstream by the gravitational field and does not present any oscillations at the top $(\theta=0)$. Such instability could not be found by a stability analysis at the north pole, but is not at the origin of droplets. When the Bond number is increased, typically above 20, a second regime has been observed. The optimal conditions correspond to interface oscillations on the upper hemicircle. The optimal algebraic gains $G_{\max }$ increase exponentially with the Bond number. The influence of the film thickness versus the curvature of the cylinder is solely contained in the modified Bond number. It is the only relevant nondimensional parameter for the oscillating regime. In effect, the Bond number may be interpreted as the ratio between the drainage time scale and the Rayleigh-Taylor instability time scale, which are the two competing mechanisms at play.

The oscillating perturbations found by adjoint-based optimization agree very well with the ones recovered semianalytically at $\theta=0$. The optimal conditions, namely the gain $G_{\max }$, the wave number $k_{\max }$, and the time to attain the largest growth are in excellent agreement. More precisely, the same exponential dependence of the maximal optimal gain and the power-law increase of the corresponding time on the Bond number have been observed. The optimal perturbations found at the top, slightly modulated in space, become optimal over the entire geometry. The upper bound of the exponential increase of the optimal gain as a function of Bo, $G_{\max } \sim \exp (13 \mathrm{Bo} / 98)$, is related to the stretching effect of the flow. Using substrates with an increasing curvature might therefore stabilize thin liquid films at even higher Bond numbers.

For Bo $\gtrsim 20$, the initial linear growth of perturbations triggers nonlinear effects, eventually resulting in the formation of droplets. The practically relevant transition between transient and dripping droplets is a function of the perturbation amplitude. Given that the algebraic gain increases exponentially with the Bond number, a threshold will always exist, no matter how small the perturbations are. We illustrated this point using full two-dimensional nonlinear simulations. The experimentally observed threshold reported in Ref. [5] is not universal and is experiment dependent.

The novelty of our work lies in the investigation of an interfacial instability using an optimal transient growth analysis with an evolving base state (here the draining solution). Such an approach based on the forward and backward integration of the direct and adjoint system opens the door to the stability analysis of other unsteady free-surface flows, ranging from thin films to deforming drops and bubbles. Another natural extension of our work is concerned with the determination of a nonlinear 
optimal transient growth analysis, as reviewed by Luchini and Bottaro [31] and Kerswell et al. [32]. Finally, our current efforts are directed towards considering the full three-dimensional geometry and performing a weakly nonlinear stability analysis, enabling the exploration of the two-dimensional pattern formation, generalizing the work of Fermigier et al. [11] to curved substrates.

\section{ACKNOWLEDGMENTS}

We would like to thank Edouard Boujo and Francesco Viola for helpful discussions on the subject of adjoint-based optimization techniques as well as Mathias Nagel for the two-phase 2D Stokes solver Ulambator. This work was funded by ERC Grant No. SIMCOMICS 280117.

\section{APPENDIX A: DERIVATION OF THE LUBRICATION EQUATION}

The derivation of the model equation presented in Sec. II A is briefly outlined hereafter. Assume a thin film coating the interior of a cylinder of radius $R$ as shown in Fig. 1(a). The initial average thickness is $H_{i}^{*}$ and the resulting film aspect ratio reads $\delta=H_{i}^{*} / R$. The characteristic lengths in the radial and polar directions are $H_{i}^{*}$ and $R$, respectively. Exploiting the small aspect ratio of the film, $\delta \ll 1$, we can use a lubrication approach [19]. Mass conservation indicates that the velocity normal to the interface is much smaller than the tangential component, $v^{*} \sim \delta u^{*} \ll u^{*}$. The low Reynolds number for this flow allows the use of the Stokes equations. The momentum equation in the radial direction is therefore

$$
0=-\frac{1}{\rho} \frac{\partial p^{*}}{\partial r^{*}}-g \cos \theta
$$

and the boundary condition for the pressure is given by $p^{*}\left(R-\bar{H}^{*}\right)=p_{0}^{*}-\gamma \kappa^{*}\left(p_{0}^{*}\right.$ is the external pressure, $\gamma$ is the surface tension, and $\kappa^{*}$ is the curvature of the interface). By integrating Eq. (A1) along the radial direction and using the boundary condition, one obtains the pressure distribution $p^{*}\left(r^{*}, \theta\right)=p_{0}^{*}-\gamma \kappa^{*}+\rho g \cos \theta\left(R-\bar{H}^{*}-r^{*}\right)$. The $\theta$ component of the momentum equation reads

$$
0=-\frac{1}{\rho r} \frac{\partial p^{*}}{\partial \theta}+v\left\{\frac{\partial}{\partial r^{*}}\left[\frac{1}{r^{*}} \frac{\partial}{\partial r^{*}}\left(r^{*} u^{*}\right)\right]+\frac{1}{r^{* 2}} \frac{\partial^{2} u^{*}}{\partial \theta^{2}}\right\}+g \sin \theta .
$$

By performing the change of variable $r^{*}=R-y$, where $0 \leqslant y \leqslant \bar{H}^{*} \ll R$, the viscous term reduces to $v \partial^{2} u^{*} / \partial y^{2}$ plus terms at least an order $\delta$ smaller. Keeping the dominant order of the viscous term, as well as the surface tension and gravitational terms, Eq. (A2) with the expression for $p^{*}$ becomes

$$
0=\frac{\gamma \kappa_{\theta}^{*}}{\rho R}+\frac{g \cos \theta \bar{H}_{\theta}^{*}}{R}+v \frac{\partial^{2} u^{*}}{\partial y^{2}}+g \sin \theta .
$$

Equation (A3) can be integrated twice and considering the zero-slip boundary condition at the cylinder surface, $u^{*}(0, \theta)=0$, as well as the zero-shear stress interface, $\partial u^{*}\left(\bar{H}^{*}, \theta\right) / \partial y=0$, yields the tangential velocity component:

$$
u^{*}(y, \theta)=\left(\frac{\gamma \kappa_{\theta}^{*}}{\mu R}+\frac{\rho g \cos \theta \bar{H}_{\theta}^{*}}{\mu R}+\frac{\rho g \sin \theta}{\mu}\right)\left(\bar{H}^{*}-\frac{y}{2}\right) y .
$$

The flow rate is given by $Q(\theta)=\int_{0}^{\bar{H}^{*}} u^{*}(y, \theta) d y$. By using the local mass conservation in cylindrical coordinates $\partial \bar{H}^{*} / \partial t+R^{-1} \partial Q / \partial \theta=0$ we eventually obtain the lubrication equation:

$$
\bar{H}_{t}^{*}+\frac{1}{3 \mu R}\left[\bar{H}^{*^{3}}(\underbrace{\frac{\gamma \kappa_{\theta}^{*}}{R}}_{\mathbf{I}}+\underbrace{\frac{\rho g \cos \theta \bar{H}_{\theta}^{*}}{R}}_{\text {II }}+\underbrace{\rho g \sin \theta}_{\text {III }})\right]_{\theta}=0
$$


where the leading order curvature derivative is $\kappa_{\theta}^{*}=R^{-2}\left(\bar{H}_{\theta \theta \theta}^{*}+\bar{H}_{\theta}^{*}\right)$. The term $\mathbf{I}$ in the spatial variation of the flux corresponds to the surface tension effects, term II corresponds to the variation of the hydrostatic pressure distribution, and term III corresponds to the drainage. A more sophisticated model could be employed to consider higher order curvature terms and larger film aspect ratios, as done by Weidner et al. [33].

The film height can be nondimensionalized by the initial average film thickness $H_{i}^{*}$ and the time by the gravitational relaxation scale $\mu R /\left(\rho g H_{i}^{* 2}\right)$ so that the lubrication equation expressed with nondimensional quantities finally reads

$$
\bar{H}_{t}+\frac{1}{3}\left\{\bar{H}^{3}\left[\frac{\delta^{2}}{\mathrm{Bo}}\left(\bar{H}_{\theta \theta \theta}+\bar{H}_{\theta}\right)+\delta \bar{H}_{\theta} \cos \theta+\sin \theta\right]\right\}_{\theta}=0,
$$

where $\mathrm{Bo}=\rho g H_{i}^{*} R / \gamma$ is the modified Bond number.

\section{APPENDIX B: LINEAR OPERATOR}

The linear operator for the perturbation equation (3) in matrix form is given by

$$
\begin{aligned}
\mathbf{L}= & -\frac{1}{3}\left[-\frac{\delta^{2} H^{3}}{\mathrm{Bo}}\left(\mathbf{D}_{2}+\mathbf{D}_{4}\right)-\frac{\delta^{2} H^{2} H_{\theta}}{\mathrm{Bo}}\left(6 \mathbf{D}_{1}+3 \mathbf{D}_{3}\right)-\frac{3 \delta^{2} H^{2} H_{\theta \theta}}{\mathrm{Bo}} \mathbf{I}\right. \\
& -\frac{3 \delta^{2} H^{2} H_{\theta \theta \theta}}{\mathrm{Bo}} \mathbf{D}_{1}-\frac{3 \delta^{2} H^{2} H_{\theta \theta \theta \theta}}{\mathrm{Bo}} \mathbf{I}-\frac{6 \delta^{2} H H_{\theta}^{2}}{\mathrm{Bo}} \mathbf{I}-\frac{6 \delta^{2} H H_{\theta} H_{\theta \theta \theta}}{\mathrm{Bo}} \mathbf{I} \\
& +\delta H^{3} \sin \theta \mathbf{D}_{1}-\delta H^{3} \cos \theta \mathbf{D}_{3}+3 \delta H^{2} H_{\theta} \sin \theta \mathbf{I}-6 \delta H^{2} H_{\theta} \cos \theta \mathbf{D}_{1} \\
& -3 \delta H^{2} H_{\theta \theta} \cos \theta \mathbf{I}-6 \delta H H_{\theta}^{2} \cos \theta \mathbf{I}-3 H^{2} \cos \theta \mathbf{I}-3 H^{2} \sin \theta \mathbf{D}_{1} \\
& \left.-6 H H_{\theta} \sin \theta \mathbf{I}\right],
\end{aligned}
$$

where $\mathbf{I}$ is the identity operator and $\mathbf{D}_{i}$ with $i=1, \ldots, 4$ are the differential operators, constructed in our study using Fourier spectral methods [26]. Subscripts with $\theta$ correspond to spatial derivatives of the drainage solution $H$.

\section{APPENDIX C: DERIVATION OF THE ADJOINT FIELDS}

We provide the extended derivation of the adjoint fields. See the introductory paper by Cossu [34] for more details on the use of variational techniques for solving constrained optimization problems.

The augmented Lagrangian of our concern, consisting of the objective function $G(T)$ to be optimized and the constraints given by the evolution equation and the initial condition, reads

$$
\mathcal{L}\left(\mathbf{h}, \mathbf{h}^{\dagger}, \mathbf{h}_{0}, \mathbf{h}_{0}^{\dagger} ; T\right)=\frac{\langle\mathbf{h}(T) \mid \mathbf{h}(T)\rangle}{\left\langle\mathbf{h}_{0} \mid \mathbf{h}_{0}\right\rangle}-\int_{0}^{T}\left\langle\mathbf{h}^{\dagger} \mid \mathbf{h}_{t}+\mathbf{L h}\right\rangle d t-\left\langle\mathbf{h}_{0}^{\dagger} \mid \mathbf{h}(0)-\mathbf{h}_{0}\right\rangle .
$$

$\langle\mathbf{a} \mid \mathbf{b}\rangle=\int_{\Omega} \mathbf{a}^{T} \mathbf{b} d \theta=\mathbf{a}^{T} \mathbf{M b}$ is the Hermitian scalar product for real quantities on the geometry $\Omega=\{\theta \mid \theta \in[0,2 \pi]\}$, defined via a weight matrix $\mathbf{M}=\frac{2 \pi}{N} \mathbf{I} \in \mathbb{R}^{N \times N}$.

By setting to zero the variations of $\mathcal{L}$ with respect to both $\mathbf{h}$ and $\mathbf{h}_{0}$, one obtains the equations for the Lagrange multipliers $\mathbf{h}^{\dagger}$ and $\mathbf{h}_{0}^{\dagger}$. For the derivation, we will make use of Frechét derivatives, which for an operator $\mathbf{A}(\mathbf{s})$ are defined by (see Ref. [34])

$$
\left\langle\frac{\partial \mathbf{A}}{\partial \mathbf{s}} \mid \delta \mathbf{s}\right\rangle=\lim _{\epsilon \rightarrow 0} \frac{\mathbf{A}(\mathbf{s}+\epsilon \delta \mathbf{s})-\mathbf{A}(\mathbf{s})}{\epsilon} .
$$


The variation of $\mathcal{L}$ with respect to $\delta \mathbf{h}$ results in

$$
\begin{aligned}
\left\langle\frac{\partial \mathcal{L}}{\partial \mathbf{h}} \mid \delta \mathbf{h}\right\rangle= & 2 \frac{\langle\mathbf{h}(T) \mid \delta \mathbf{h}(T)\rangle}{\left\langle\mathbf{h}_{0} \mid \mathbf{h}_{0}\right\rangle}-\int_{0}^{T}\left\langle\mathbf{h}^{\dagger} \mid \delta \mathbf{h}_{t}+\mathbf{L} \delta \mathbf{h}\right\rangle d t-\left\langle\mathbf{h}_{0}^{\dagger} \mid \delta \mathbf{h}(0)\right\rangle \\
= & 2 \frac{\langle\mathbf{h}(T) \mid \delta \mathbf{h}(T)\rangle}{\left\langle\mathbf{h}_{0} \mid \mathbf{h}_{0}\right\rangle}-\int_{0}^{T}\left\langle\mathbf{h}^{\dagger} \mid \delta \mathbf{h}_{t}\right\rangle d t-\int_{0}^{T}\left\langle\mathbf{h}^{\dagger} \mid \mathbf{L} \delta \mathbf{h}\right\rangle d t-\left\langle\mathbf{h}_{0}^{\dagger} \mid \delta \mathbf{h}(0)\right\rangle \\
= & 2 \frac{\langle\mathbf{h}(T) \mid \delta \mathbf{h}(T)\rangle}{\left\langle\mathbf{h}_{0} \mid \mathbf{h}_{0}\right\rangle}-\left\langle\mathbf{h}^{\dagger}(T) \mid \delta \mathbf{h}(T)\right\rangle+\left\langle\mathbf{h}^{\dagger}(0) \mid \delta \mathbf{h}(0)\right\rangle+\int_{0}^{T}\left\langle\mathbf{h}_{t}^{\dagger} \mid \delta \mathbf{h}\right\rangle d t \\
& -\int_{0}^{T}\left\langle\mathbf{L}^{\dagger} \mathbf{h}^{\dagger} \mid \delta \mathbf{h}\right\rangle d t-\left\langle\mathbf{h}_{0}^{\dagger} \mid \delta \mathbf{h}(0)\right\rangle \\
= & \left\langle 2 \frac{\mathbf{h}(T)}{\left\langle\mathbf{h}_{0} \mid \mathbf{h}_{0}\right\rangle}-\mathbf{h}^{\dagger}(T) \mid \delta \mathbf{h}(T)\right\rangle+\int_{0}^{T}\left\langle\mathbf{h}_{t}^{\dagger}-\mathbf{L}^{\dagger} \mathbf{h}^{\dagger} \mid \delta \mathbf{h}\right\rangle d t+\left\langle\mathbf{h}^{\dagger}(0)-\mathbf{h}_{0}^{\dagger} \mid \delta \mathbf{h}(0)\right\rangle .
\end{aligned}
$$

The distributive property of the scalar product has been used in Eq. (C4), integration by part in Eq. (C5), and the definition of the adjoint operator, namely $\langle\mathbf{a} \mid \mathbf{L b}\rangle=\left\langle\mathbf{L}^{\dagger} \mathbf{a} \mid \mathbf{b}\right\rangle$, in Eq. (C6). Since the Hermitian scalar product is defined with an identity (up to a factor) matrix $\mathbf{M}$, the discrete adjoint corresponds to the transpose of the direct one: $\mathbf{L}^{\dagger}=\mathbf{L}^{T}$, where $\mathbf{L}$ is defined by Eq. (B1). The analytical calculation of the continuous adjoint operator can therefore be avoided [23]. Similarly, by imposing a vanishing variation of $\mathcal{L}$ to any $\delta \mathbf{h}$, one eventually obtains

$$
\begin{aligned}
\left\langle\frac{\partial \mathcal{L}}{\partial \mathbf{h}} \mid \delta \mathbf{h}\right\rangle=0, \quad \forall \delta \mathbf{h} \Leftrightarrow & \mathbf{h}^{\dagger}(T)=2 \frac{\mathbf{h}(T)}{\left\langle\mathbf{h}_{0} \mid \mathbf{h}_{0}\right\rangle}, \\
& \mathbf{h}_{t}^{\dagger}-\mathbf{L}^{\dagger} \mathbf{h}^{\dagger}=\mathbf{0} \quad t \in[0, T], \\
& \mathbf{h}_{0}^{\dagger}=\mathbf{h}^{\dagger}(0) .
\end{aligned}
$$

These are the terminal conditions for the adjoint field, Eq. (8), the adjoint equation (9), and the definition of the second adjoint variable, Eq. (10), respectively. The variation of the augmented Lagrangian with respect to $\mathbf{h}_{0}$ reads

$$
\begin{aligned}
\left\langle\frac{\partial \mathcal{L}}{\partial \mathbf{h}_{0}} \mid \delta \mathbf{h}_{0}\right\rangle & =\left\langle-2 \mathbf{h}_{0} \frac{\langle\mathbf{h}(T) \mid \mathbf{h}(T)\rangle}{\left\langle\mathbf{h}_{0} \mid \mathbf{h}_{0}\right\rangle^{2}} \mid \delta \mathbf{h}_{0}\right\rangle-\int_{0}^{T}\left\langle\mathbf{h}^{\dagger} \mid \mathbf{0}\right\rangle d t-\left\langle\mathbf{h}_{0}^{\dagger} \mid-\delta \mathbf{h}_{0}\right\rangle \\
& =\left\langle-2 \mathbf{h}_{0} \frac{\langle\mathbf{h}(T) \mid \mathbf{h}(T)\rangle}{\left\langle\mathbf{h}_{0} \mid \mathbf{h}_{0}\right\rangle^{2}}+\mathbf{h}_{0}^{\dagger} \mid \delta \mathbf{h}_{0}\right\rangle .
\end{aligned}
$$

The stationarity condition with respect to any $\delta \mathbf{h}_{0}$ yields the optimality condition, Eq. (11):

$$
\left\langle\frac{\partial \mathcal{L}}{\partial \mathbf{h}_{0}} \mid \delta \mathbf{h}_{0}\right\rangle=0, \quad \forall \delta \mathbf{h}_{0} \Leftrightarrow \mathbf{h}_{0}=\mathbf{h}_{0}^{\dagger} \frac{\left\langle\mathbf{h}_{0} \mid \mathbf{h}_{0}\right\rangle^{2}}{2\langle\mathbf{h}(T) \mid \mathbf{h}(T)\rangle} .
$$

\section{APPENDIX D: NUMERICAL METHODS}

The developed numerical schemes are validated hereafter. For an initially uniform film, the analytical solution of the lubrication equation (1) at $\theta=0$ is given by $(1+2 t / 3)^{-1 / 2}$; see for example Refs. [5,20]. The numerical solution of Eq. (1) with $N=256$ collocation points nicely agrees with the analytical one [see Fig. 13(a)]. The comparison of the numerical solution of the lubrication equation to the analytical solution allows us to verify the absence of transient waves in the base state (draining) solution. Also, as discussed in Sec. II A, the effect of the Bond number on the base state solution is only visible in the meniscus connecting the draining film to the liquid pool al the bottom of the cylinder. When Bo $\gtrsim 350$, the amplification of numerical errors results in the formation of droplets at the top of the geometry, which allows us to clearly distinguish between 


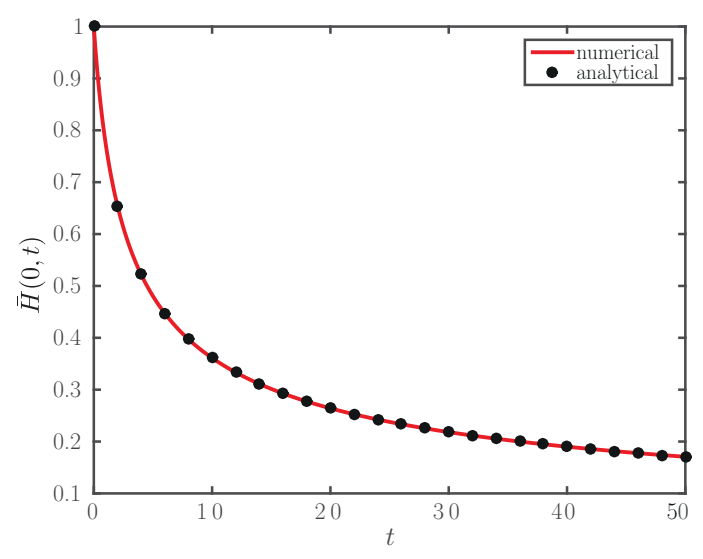

(a)

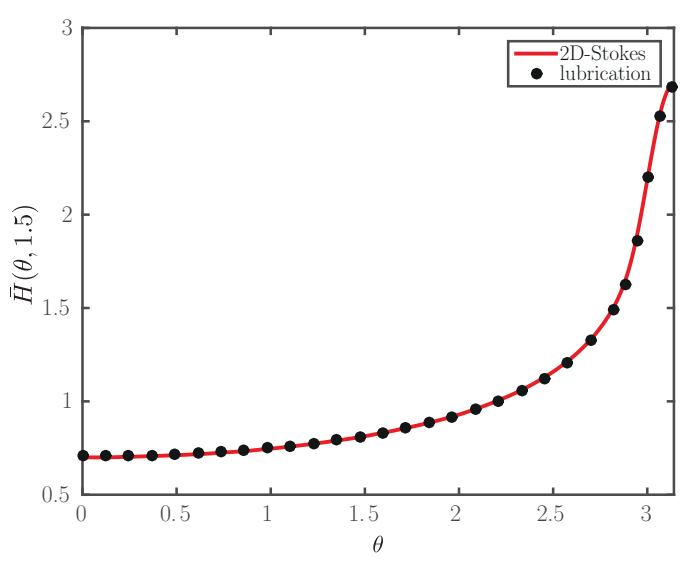

(b)

FIG. 13. (a) Comparison between the numerical (solid line) and analytical (bullets) solutions of the lubrication equation (1) for the film thickness at the north pole, $\theta=0$. (b) Comparison between the film-thickness profile obtained by the resolution of the lubrication equation (1) (bullets) and using the two-phase 2D Stokes solver Ulambator (solid line) at $t=1.5$. Bo $=20, \delta=10^{-2}$, and $N=256$.

a correct base state and a base state containing transient waves. For $\theta>0$, the numerical result is compared to the two-phase 2D Stokes simulation result obtained with Ulambator [28]. The good correspondence is visible in Fig. 13(b).

A convergence study for the drainage solution and for the transient growth gain is presented in Fig. 14. Convergence is attained already for $N=256$ collocation points for $\mathrm{Bo}=5$. Note that the capillary length, expressed with nondimensional quantities as $\ell_{c}=1 /(\operatorname{Bo} \delta)$, has to be resolved. The condition $\ell_{c}>\Delta x=2 \pi /(N \delta)$ imposes a large number of discretization points $N$ for large Bond numbers, the reason why our numerical study is restricted to Bo $\leqslant 80$.

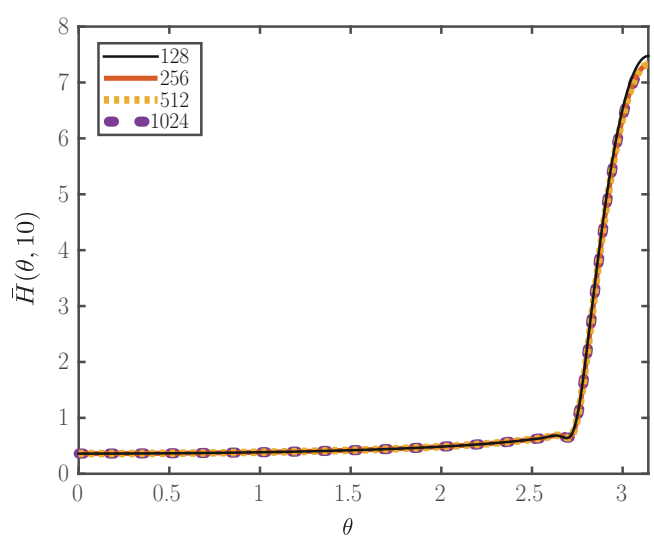

(a)

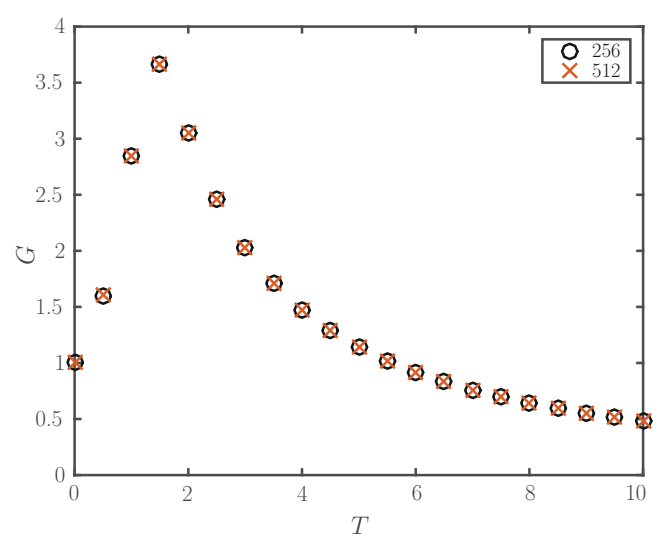

(b)

FIG. 14. Convergence study for the resolution of (a) the lubrication equation (1) for the film thickness profile at $t=10$ and (b) the transient growth gain for $\mathrm{Bo}=5$ and $\delta=10^{-2}$. Legend: number of grid points $N$. 
[1] L. Rayleigh, Proc. London Math. Soc. S1-14, 170 (1882).

[2] G. Taylor, Proc. R. Soc. London, Ser. A 201, 192 (1950).

[3] D. H. Sharp, Phys. D (Amsterdam, Neth.) 12, 3 (1984).

[4] R. Kaita, L. Berzak, D. Boyle, T. Gray, E. Granstedt, G. Hammett, C. M. Jacobson, A. Jones, T. Kozub, H. Kugel et al., Fusion Eng. Des. 85, 874 (2010).

[5] P. H. Trinh, H. Kim, N. Hammoud, P. D. Howell, S. J. Chapman, and H. A. Stone, Phys. Fluids 26, 051704 (2014).

[6] A. Lee, P.-T. Brun, J. Marthelot, G. Balestra, F. Gallaire, and P. M. Reis, Nat. Commun. 7, 11155 (2016).

[7] V. Lapuerta, F. J. Mancebo, and J. M. Vega, Phys. Rev. E 64, 016318 (2001).

[8] A. Alexeev and A. Oron, Phys. Fluids 19, 082101 (2007).

[9] D. E. Weidner, L. W. Schwartz, and M. H. Eres, Chem. Product Process Model. 2, 19 (2007).

[10] R. Cimpeanu, D. T. Papageorgiou, and P. G. Petropoulos, Phys. Fluids 26, 022105 (2014).

[11] M. Fermigier, L. Limat, J. Wesfreid, P. Boudinet, and C. Quilliet, J. Fluid Mech. 236, 349 (1992).

[12] L. Limat, P. Jenffer, B. Dagens, E. Touron, M. Fermigier, and J. Wesfreid, Phys. D (Amsterdam, Neth.) 61, 166 (1992).

[13] J. R. Lister, J. M. Rallison, and S. J. Rees, J. Fluid Mech. 647, 239 (2010).

[14] P.-T. Brun, A. Damiano, P. Rieu, G. Balestra, and F. Gallaire, Phys. Fluids 27, 084107 (2015).

[15] B. Scheid, N. Kofman, and W. Rohlfs, Phys. Fluids 28, 044107 (2016).

[16] P. J. Schmid, Annu. Rev. Fluid Mech. 39, 129 (2007).

[17] A. L. Bertozzi and M. P. Brenner, Phys. Fluids 9, 530 (1997).

[18] G. Gallino, L. Zhu, and F. Gallaire, J. Fluid Mech. 786, R2 (2016).

[19] A. Oron, S. H. Davis, and S. G. Bankoff, Rev. Mod. Phys. 69, 931 (1997).

[20] D. Takagi and H. E. Huppert, J. Fluid Mech. 647, 221 (2010).

[21] Y. Couder, E. Fort, C.-H. Gautier, and A. Boudaoud, Phys. Rev. Lett. 94, 177801 (2005).

[22] P. J. Olsson and D. S. Henningson, Stud. Appl. Math. 94, 183 (1995).

[23] P. J. Schmid and D. S. Henningson, Stability and Transition in Shear Flows, Applied Mathematical Sciences Vol. 142 (Springer-Verlag, New York, 2001).

[24] D. Barkley, H. Blackburn, and S. J. Sherwin, Int. J. Num. Meth. Fluids 57, 1435 (2008).

[25] X. Mao, H. M. Blackburn, and S. J. Sherwin, J. Comput. Phys. 235, 258 (2013).

[26] J. A. Weideman and S. C. Reddy, ACM Trans. Math. Software 26, 465 (2000).

[27] L. N. Trefethen, Spectral Methods in MATLAB, Vol. 10 (Siam, Philadelphia, 2000).

[28] M. Nagel and F. Gallaire, Comp. Fluids 107, 272 (2015).

[29] E. Villermaux, C. R. Mec. 340, 555 (2012).

[30] J. Eggers and E. Villermaux, Rep. Prog. Phys. 71, 036601 (2008).

[31] P. Luchini and A. Bottaro, Annu. Rev. Fluid Mech. 46, 493 (2014).

[32] R. Kerswell, C. Pringle, and A. Willis, Rep. Prog. Phys. 77, 085901 (2014).

[33] D. E. Weidner, L. W. Schwartz, and M. H. Eres, J. Colloid Interface Sci. 187, 243 (1997).

[34] C. Cossu, Appl. Mech. Rev. 66, 024801 (2014). 